\title{
ON ABSTRACT COMMENSURATORS OF SURFACE GROUPS
}

\author{
KHALID BOU-RABEE AND DANIEL STUDENMUND
}

\begin{abstract}
Let $\Gamma$ be the fundamental group of a surface of finite type and $\operatorname{Comm}(\Gamma)$ be its abstract commensurator. Then $\operatorname{Comm}(\Gamma)$ contains the solvable Baumslag-Solitar groups $\left\langle a, b: a b a^{-1}=b^{n}\right\rangle$ for any $n>1$. Moreover, the Baumslag-Solitar group $\left\langle a, b: a b^{2} a^{-1}=b^{3}\right\rangle$ has an image in $\operatorname{Comm}(\Gamma)$ that is not residually finite. Our proofs are computer-assisted.

Our results also illustrate that finitely-generated subgroups of $\operatorname{Comm}(\Gamma)$ are concrete objects amenable to computational methods. For example, we give a proof that $\left\langle a, b: a b^{2} a^{-1}=b^{3}\right\rangle$ is not residually finite without the use of normal forms of HNN extensions.
\end{abstract}

\section{INTRODUCTION}

Let $G$ be a group. The abstract commensurator ${ }^{1}$ of $G$, denoted $\operatorname{Comm}(G)$, is the set of equivalence classes of isomorphisms $\phi: H_{1} \rightarrow H_{2}$ for finite-index subgroups $H_{1}, H_{2} \leq G$, where two isomorphisms are equivalent if they are both defined and equal on a common finite-index subgroup of $G$. The set $\operatorname{Comm}(G)$ is a group under composition. Elements of $\operatorname{Comm}(G)$ are called commensurators of $G$.

The results of this paper serve two purposes. First, they shed light on the structure of $\operatorname{Comm}(\Gamma)$ when $\Gamma$ is the fundamental group of a surface of finite type. These abstract commensurator groups are not well-understood, and in particular are known to be neither finitely generated [BB10] nor linear over $\mathbb{C}$ [Stu15, Proposition 7.6]. Second, the methods of proof suggest that maps into abstract commensurators of fundamental groups of surfaces of finite type provide a new sort of "representation theory" of groups that have no faithful representations through matrices. This view has immediate utility: we give a new and concrete proof of a classical result of Baumslag-Solitar concerning their one-relator groups BS62.

1.1. The main result. The Baumslag-Solitar groups are given by the presentation

$$
\operatorname{BS}(m, n):=\left\langle a, b: a b^{m} a^{-1}=b^{n}\right\rangle .
$$

By surface group, we mean the fundamental group of a surface with finite genus, and finitely many punctures and boundary components. Any surface group is finitely generated.

Theorem 1. Let $\Gamma$ be a surface group, and let $n>1$. Then the group $\operatorname{Comm}(\Gamma)$ contains $\operatorname{BS}(1, n)$. Moreover, $\operatorname{Comm}(\Gamma)$ contains a non-residually finite image of $\mathrm{BS}(2,3)$.

Date: March 8, 2019.

2000 Mathematics Subject Classification. Primary 20E26, 20B07; Secondary 20K10.

Key words and phrases. commensurators, algebraic groups, residually finite groups.

DS supported in part by NSF Grant \#1547292.

${ }^{1}$ In the literature this is also known as the virtual automorphism group. 
Our proof of Theorem 1 appears in $\$ 2$ with supplementary code and theory appearing in the appendix. Since $\operatorname{Comm}(G)$ is an invariant of the commensurability class of $G$, Theorem 1 is a statement about the abstract commensurators of two groups: the nonabelian free group of rank two, and the fundamental group of a compact surface of genus two.

We prove the nonabelian free group case first, and then build upon it to handle the compact case. The first part of each case uses some of the elementary theory of surface topology. The second part of each case requires some computer-assistance, using the GAP System for Computational Discrete Algebra [GAP17.

The length of our proof belies the difficulty of the proof of the cocompact case, which relies on a long and delicate computation supplied in Appendix C. The appendix is a substantial part of the theory in this paper: the setup, hand computations, and computer computations find an explicit representation of a Baumslag-Solitar group in $\operatorname{Comm}(\Gamma)$.

1.2. On local residual finiteness. A group is (locally) residually finite if every (finitely generated) subgroup is residually finite. Linear groups (e.g., fundamental groups of surfaces, $\mathrm{GL}_{n}(\mathbb{C})$, or $\mathrm{GL}_{n}\left(\mathbb{F}_{p}[t]\right)$ ) and mapping class groups of surfaces (e.g., braid groups) are important classes of locally residually finite groups [Mal56, Gro75.

Our main theorem determines precisely when the abstract commensurator of a lattice in a semisimple Lie group is locally residually finite. See $\$ 3$ for the proof.

Corollary 2. Let $\Gamma$ be an irreducible lattice in a semisimple Lie group $G$ without compact factors. Then $\operatorname{Comm}(\Gamma)$ is locally residually finite if and only if $G$ is not locally isomorphic to $\mathrm{PSL}_{2}(\mathbb{R})$. In particular, the abstract commensurator of any lattice in $\mathrm{PSL}_{n}(\mathbb{R})$ is locally residually finite if and only if $n \neq 2$.

The fact that lattices in $\mathrm{PSL}_{2}(\mathbb{R})$ have abstract commensurators which are not locally residually finite is surprising for two reasons. First, Baumslag showed that for any finitely-generated residually finite $G$, the group $\operatorname{Aut}(G)$ (which $\operatorname{Comm}(G)$ can be thought to generalize) is residually finite [Bau63. Second, Odden showed that the abstract commensurator of the fundamental group of a closed surface is a mapping class group of an inverse limit of surfaces (the "universal hyperbolic solenoid") Odd05.

1.3. On residual finiteness of $\mathrm{BS}(2,3)$. Our proof of Theorem 1 explicitly identifies an element $\gamma \in \mathrm{BS}(2,3)$ in the kernel of a surjective homomorphism $\operatorname{BS}(2,3) \rightarrow$ $\mathrm{BS}(2,3)$, then concretely shows that $\gamma$ has nontrivial image under a homomorphism $\mathrm{BS}(2,3) \rightarrow \operatorname{Comm}(\Gamma)$. Thus, our methods prove that $\mathrm{BS}(2,3)$ is not residually finite without using the normal forms ensured by the Britton's Lemma Bri63. Bypassing this step makes the original proof that $\operatorname{BS}(2,3)$ is not residually finite, due to Baumslag-Solitar BS62, elementary and concrete. We emphasize that our proof relies solely on the $\operatorname{Comm}\left(F_{2}\right)$ case, and hence does not require computer verification. See $\$ 4$ for the proof.

Theorem 3 ([BS62]). The group BS(2,3) is not residually finite.

1.4. On two groups. Let $F_{2}$ be the nonabelian free group of rank two. Let $\Gamma_{2}$ be the fundamental group of a compact surface of genus two. The groups $\operatorname{Comm}\left(F_{2}\right)$ and $\operatorname{Comm}\left(\Gamma_{2}\right)$ share a number of properties: Bartholdi-Bogopolski showed that neither group is finitely generated [BB10]. Moreover, finitely-generated subgroups 
of them have solvable word problem (see \$1.5). The proof of Theorem 1 shows that they both contain many infinite images of Baumslag-Solitar groups.

In spite of these similarities, in $\$ 5$ we show that $\operatorname{Comm}\left(F_{2}\right)$ contains more finite subgroups than $\operatorname{Comm}\left(\Gamma_{2}\right)$ to prove that they are not isomorphic.

Proposition 4. The groups $\operatorname{Comm}\left(F_{2}\right)$ and $\operatorname{Comm}\left(\Gamma_{2}\right)$ are not isomorphic.

1.5. On the word problem. Although the groups $\operatorname{Comm}(\Gamma)$ are not well-understood when $\Gamma$ is a surface group, their finitely-generated subgroups may be understood concretely, as in the computations that appear in Appendix B In particular, the word problem is solvable. A more general version of the following is proved in $\$ 6$

Proposition 5. Finitely-generated subgroups of abstract commensurators of surface groups have solvable word problem.

Theorem 1 provides an example of a non-residually finite group $G$ that faithfully embeds in $\operatorname{Comm}\left(F_{2}\right)$. Such $G$ cannot be completely understood through its linear representations, while words in its image in $\operatorname{Comm}\left(F_{2}\right)$ can be evaluated by a computer. This shows that representations into $\operatorname{Comm}\left(F_{2}\right)$ may be useful for understanding abstract groups without faithful linear representations. See $\$ 6$ for further discussion and questions.

1.6. Historical remarks. Questions about abstract commensurators of surface groups have been asked for at least 30 years [Man87. More generally, abstract commensurators detect arithmeticity of lattices in higher-rank semisimple Lie groups (see discussion after Corollary 2 in $\$ 1.2$, give "generalized Hecke operators" from number theory (see [Sha00, page 10]), play a role in extending linear group methods to nonlinear settings (e.g., with mapping class groups [FH07, §1]), and are used to give more algebraic descriptions of existing infinite finitely presented simple groups (e.g., [R0̈2]).

Abstract commensurators can also be understood in the context of coarse geometry. The quasi-isometry group $\mathrm{QI}(\Gamma)$ of a group $\Gamma$ is the group of equivalences classes of self-quasi-isometries $f: \Gamma \rightarrow \Gamma$, up to coarse equivalence. There is a natural map $\operatorname{Comm}(\Gamma) \rightarrow \mathrm{QI}(\Gamma)$ which is injective when $\Gamma$ is finitely generated [FM02, Theorem 7.4]. This shows, for example, that the abstract commensurator of a word hyperbolic group acts faithfully on its Gromov boundary.

That the abstract commensurator of a free group of rank two is not locally residually finite is a well-known folklore result. The folklore proof relies on the existence of a simple, finitely presented group that is isomorphic to an amalgamated product of two finitely-generated free groups over a common finite-index subgroup. The first groups of this kind were constructed by Burger-Mozes [BM97]. It is unknown whether any of the Burger-Mozes groups embed inside the abstract commensurator of a closed oriented surface group of genus two.

Acknowledgements. We are grateful to Benson Farb and Andrew Putman for conversations and support. We are especially grateful to Yves Cornulier and Benson Farb for giving us many useful comments that substantially improved the paper.

\section{Proof of Theorem 1}

Let $F_{2}$ be the free group of rank two. Let $\Sigma_{g}$ be the compact oriented surface of genus $g$. Let $\Gamma$ be a surface group. As stated in $\$ 1.1$ and $\$ 1.4$, the group 
$\operatorname{Comm}(\Gamma)$ is isomorphic to either $\operatorname{Comm}\left(F_{2}\right)$ or $\operatorname{Comm}\left(\pi_{1}\left(\Sigma_{2}, *\right)\right)$. We handle each case separately, as they are different enough to warrant different proofs (the first serving as an outline of the second).

It will be important to us in each case that $\Gamma$ satisfies the unique root property: if elements $x, y \in \Gamma$ satisfy $x^{n}=y^{n}$ for some $n \neq 0$, then $x=y$ BB10, Lemma 2.2], [BH99, pages 462-463]. A consequence of the unique root property is that two isomorphisms $f: A \rightarrow B$ and $g: C \rightarrow D$ between finite-index subgroups of $\Gamma$ represent the same element of $\operatorname{Comm}(\Gamma)$ if and only if $\left.f\right|_{A \cap C}=\left.g\right|_{A \cap C}$. In particular, to check that $f$ represents a nontrivial elements of $\operatorname{Comm}(\Gamma)$, it suffices to find an element $\gamma \in \Gamma$ such that $f(\gamma) \neq \gamma$.

2.1. The $\operatorname{Comm}\left(F_{2}\right)$ case. We first describe a method for constructing images of $\operatorname{BS}(n, m)$ in $\operatorname{Comm}\left(F_{2}\right)$. Set $F_{2}=\langle A, B\rangle$. Let $\pi_{1}: F_{2} \rightarrow \mathbb{Z} / m \times \mathbb{Z} / n$ be the map given by $A \mapsto(1,0)$ and $B \mapsto(0,1)$. Let $\pi_{2}: F_{2} \rightarrow \mathbb{Z} / m \times \mathbb{Z} / n$ be the map given by $A \mapsto(0,1)$ and $B \mapsto(1,0)$. Set $\Delta_{1}=\operatorname{ker}\left(\pi_{1}\right)$ and $\Delta_{2}=\operatorname{ker}\left(\pi_{2}\right)$.

Let $\phi$ be the commensurator with representative $f: F_{2} \rightarrow F_{2}$ given by $f(\gamma)=$ $A \gamma A^{-1}$. Let $\psi$ be a commensurator with representative $g: \Delta_{1} \rightarrow \Delta_{2}$, where $g\left(A^{m}\right)=A^{n}$; such an isomorphism $g$ exists because $\Delta_{1}$ and $\Delta_{2}$ are free groups of the same rank in which $A^{m}$ and $A^{n}$, respectively, are elements in free generating sets. Then the commensurator $\psi \circ \phi^{m} \circ \psi^{-1}$ has a representative $h=g \circ f^{m} \circ g^{-1}$ such that for every $\gamma \in \Delta_{2}$,

$$
h(\gamma)=g \circ f^{m} \circ g^{-1}(\gamma)=g\left(A^{m} g^{-1}(\gamma) A^{-m}\right)=A^{n}\left(g \circ g^{-1}(\gamma)\right) A^{-n}=A^{n} \gamma A^{-n},
$$

and hence $\psi \circ \phi^{m} \circ \psi^{-1}=\phi^{n}$. Thus, the assignment $a \mapsto \psi$ and $b \mapsto \phi$ defines a homomorphism $\Phi: \operatorname{BS}(m, n) \rightarrow \operatorname{Comm}\left(F_{2}\right)$.

Next, we show that the map $\operatorname{BS}(m, n) \rightarrow\langle\psi, \phi\rangle$ is an isomorphism of groups when $m=1$. Let $z$ be in the kernel of this map. Then $z=a^{s} \gamma$, where $\gamma$ is in the normal closure of $b$. Since $\langle\langle b\rangle\rangle=\left\langle a^{t} b a^{-t}: t \leq 0\right\rangle$, we have that $z$ is conjugate to an element of the form $a^{s} b^{t}$ for some integers $s$ and $t$. It suffices to show that $\psi^{s} \circ \phi^{t}$ is only trivial in $\operatorname{Comm}\left(F_{2}\right)$ if $s=t=0$.

If $s>0$ then any $\psi^{s}$ has a representative that maps $A$ to $A^{n^{s}}$. By the unique root property of $F_{2}$, it is impossible for an automorphism of $F_{2}$ to send $A$ to $A^{n^{s}}$. Similarly, if $s<0$ then $\psi^{s}$ has a representative that maps $A^{n^{-s}}$ to $A$, which cannot be extended to an automorphism of $F_{2}$. If $\psi^{s} \circ \phi^{t}$ is trivial, then $\psi^{s}$ has a representative that is an automorphism of $F_{2}$, and so $s=0$. It is clear that $\phi^{t}$ is trivial only if $t=0$ because $f^{t}\left(B^{\ell}\right)=A^{t} B^{\ell} A^{-t}$ for all $\ell$. This complete the proof of the $m=1$ case.

To finish, we need to show that when $m=2$ and $n=3$, the image of $\operatorname{BS}(m, n)$ as defined above is not residually finite. To do this, we need to show that element

$$
\gamma:=b^{-1} a b a^{-1} b^{-1} a b a^{-1} b^{-1}
$$

has nontrivial image. Indeed, it is in the kernel of the surjective map $\mathrm{BS}(2,3) \rightarrow$ $\mathrm{BS}(2,3)$ given by $a \mapsto a$ and $b \mapsto b^{2}$, and thus is in the residual finiteness kernel of $\mathrm{BS}(2,3)$. See $\$ 4$ for more details.

The proof is completed by evaluating the commensurator $\Phi(\gamma)$ on the word $B A B^{-1} A^{-1} \in F_{2}$ and checking that the result is not equal to $B A B^{-1} A^{-1}$. This is straightforward, but tedious, to compute by hand. To perform this calculation, we used computer assistance. See the code in Appendix B and explanations there to end the proof of the $\operatorname{Comm}\left(F_{2}\right)$ case. 


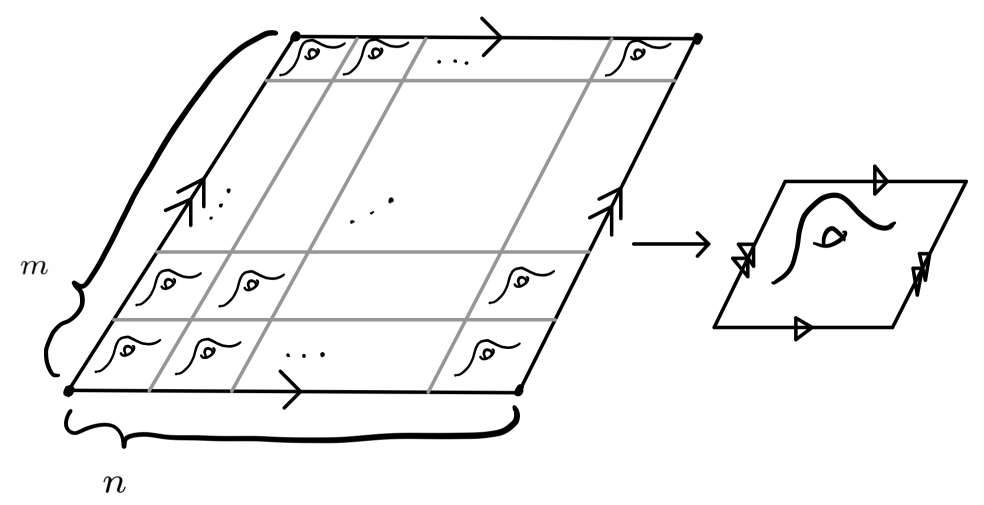

Figure 1. Cover of $\Sigma_{2}$ for $\$ 2.2$

2.2. The $\operatorname{Comm}\left(\pi_{1}\left(\Sigma_{2}, *\right)\right)$ case. As in $\$ 2.1$, we begin by describing how to obtain images of $\operatorname{BS}(n, m)$ inside $\operatorname{Comm}\left(\pi_{1}\left(\Sigma_{2}, *\right)\right)$. Let $[X, Y]:=X Y X^{-1} Y^{-1}$ and let

$$
\Gamma_{2}:=\pi_{1}\left(\Sigma_{2}, *\right)=\langle A, B, C, D:[A, B][C, D]\rangle .
$$

Set $\pi_{1}: \Gamma_{2} \rightarrow \mathbb{Z} / m \times \mathbb{Z} / n$ be a map satisfying $A \mapsto(1,0)$, where in the cover corresponding to ker $\pi_{1}$ the curve corresponding to $A^{m}$ lifts to a non-separating simple closed curve. See Figure 1 for the cover corresponding to an example of such a map. Similarly, set $\pi_{2}: \Gamma_{2} \rightarrow \mathbb{Z} / m \times \mathbb{Z} / n$ be a map satisfying $A \mapsto(0,1)$, where in the cover corresponding to ker $\pi_{1}$ the curve corresponding to $A^{n}$ lifts to a non-separating simple closed curve. Set $\Delta_{1}=\operatorname{ker}\left(\pi_{1}\right)$ and $\Delta_{2}=\operatorname{ker}\left(\pi_{2}\right)$.

Let $\phi$ be the commensurator with representative $f: \Gamma_{2} \rightarrow \Gamma_{2}$ given by $f(\gamma)=$ $A \gamma A^{-1}$. To define the commensurator $\psi$ we need some additional setup:

Let $p_{1}: S_{1} \rightarrow \Sigma_{2}$ and $p_{2}: S_{2} \rightarrow \Sigma_{2}$ be the covers corresponding to $\Delta_{1}$ and $\Delta_{2}$ respectively. Then $A^{m}$ and $A^{n}$ lift to non-separating simple-closed curves in $S_{1}$ and $S_{2}$ by construction. Any oriented surface group is uniquely determined by its Euler characteristic. Both $S_{1}$ and $S_{2}$ cover $\Sigma_{2}$ with degree $m n$ and hence have Euler characteristic $-2 m n$ by the Riemann-Hurwitz formula. It follows that $S_{1}$ and $S_{2}$ are homeomorphic. Moreover, by the Change of Coordinates Principle [FM12, p. 37] there i: ${ }^{2}$ a homeomorphism $S_{1} \rightarrow S_{2}$ inducing an isomorphism $g: \Delta_{1} \rightarrow \Delta_{2}$, where $g\left(A^{m}\right)=A^{n}$. Let $\psi$ be the commensurator with representative $g$. Then the commensurator $\psi \circ \phi^{n} \circ \psi^{-1}$ has a representative $h=g \circ f^{n} \circ g^{-1}$ such that for every $\gamma \in \Delta_{2}$,

$$
h(\gamma)=g \circ f^{m} \circ g^{-1}(\gamma)=g\left(A^{m} g^{-1}(\gamma) A^{-m}\right)=A^{n}\left(g \circ g^{-1}(\gamma)\right) A^{-n}=A^{n} \gamma A^{-n},
$$

and hence $\psi \circ \phi^{m} \circ \psi^{-1}=\phi^{n}$. Thus, the assignment $a \mapsto \psi$ and $b \mapsto \phi$ defines a homomorphism $\Phi: \operatorname{BS}(m, n) \rightarrow \operatorname{Comm}\left(\Gamma_{2}\right)$.

Since $\Gamma_{2}$ has the unique root property, the argument for showing that the map $\operatorname{BS}(m, n) \rightarrow\langle\psi, \phi\rangle$ is an isomorphism when $m=1$ in $\$ 2.1$ applies here verbatim. Hence, it only remains to show that when $m=2$ and $n=3$ the element

$$
\gamma:=b^{-1} a b a^{-1} b^{-1} a b a^{-1} b^{-1}
$$

\footnotetext{
${ }^{2}$ See Appendix $\mathrm{C}$ for an explicit construction of such a map in the case $(m, n)=(2,3)$.
} 
has nontrivial image, since this is in the residual finiteness kernel of $\operatorname{BS}(m, n)$. The rest of the proof, as before, is computer-assisted. The computer computations are more difficult to implement here as surface groups do not have as much flexibility as free groups. See Appendix $\mathrm{C}$ and explanations there to end the proof.

\section{Proof of Corollary 2}

By Theorem 1 it suffices to show that the abstract commensurator of any irreducible lattice $\Lambda$ in a semisimple Lie group $G$ without compact factors and not locally isomorphic to $\mathrm{PSL}_{2}(\mathbb{R})$ is locally residually finite. Indeed, for any such $\Lambda \leq G$, strong rigidity results of Mostow-Prasad-Margulis Mar91] show that every commensurator of $\Lambda$ extends to an automorphism of $G$. By the Borel density theorem Bor60, the induced map $\operatorname{Comm}(\Lambda) \rightarrow \operatorname{Aut}(G)$ is faithful. This shows $\operatorname{Comm}(\Lambda)$ is linear and thus locally residually finite by Malćev's Theorem Mal71.

\section{Proof of Theorem 3}

In $\$ 2.1$ there is a concrete and elementary proof that the element

$$
\gamma:=b^{-1} a b a^{-1} b^{-1} a b a^{-1} b^{-1}
$$

is not the identity in $B S(2,3)$. We emphasize that this proof does not require a computer (although it is comforting that the computation can be checked using one). The salient property of this element is that it is in the kernel of the surjective map $\rho: \mathrm{BS}(2,3) \rightarrow \mathrm{BS}(2,3)$ given by $a \mapsto a$ and $b \mapsto b^{2}$. It is a standard argument that this shows $\mathrm{BS}(2,3)$ is not residually finite. We include it here for completeness:

If $\operatorname{BS}(2,3)$ were residually finite, then there would exist a finite group $Q$ and a homomorphism $f: \operatorname{BS}(2,3) \rightarrow Q$ such that $f(\gamma) \neq 1$. For each $k$ there is an element $\gamma_{k}$ such that $\rho^{k}\left(\gamma_{k}\right)=\gamma$. Then $\gamma_{k}$ is not in the kernel of $f \circ \rho^{k}$, but lies in the kernel of $f \circ \rho^{n}$ for all $n \geq k$, and hence the maps $f \circ \rho^{k}: \operatorname{BS}(2,3) \rightarrow Q$ are all distinct for positive $k$. This is impossible since there are only finitely many maps from a finitely-generated group to a fixed finite group.

\section{Proof of Proposition 4}

We will show that the group $\operatorname{Comm}\left(F_{2}\right)$ contains an infinite collection of groups not in $\operatorname{Comm}\left(\Gamma_{2}\right)$. To start, we record that $\operatorname{Comm}\left(F_{2}\right)$ contains every finite group. Every finitely-generated free group $F_{n}$ for $n \geq 2$ is a finite-index subgroup of $F_{2}$, and because $F_{2}$ has the unique root property, the natural maps $\operatorname{Aut}\left(F_{n}\right) \rightarrow \operatorname{Comm}\left(F_{2}\right)$ are injective. Every finite group lies in some symmetric group, hence in $\operatorname{Aut}\left(F_{n}\right)$ for some $n$.

However, $\operatorname{Comm}\left(\Gamma_{2}\right)$ only contains finite groups that have a cyclic subgroup of index at most 2. Indeed, the Gromov boundary of $\Gamma_{2}$ is $S^{1}$, and so $\operatorname{Comm}\left(\Gamma_{2}\right)$ acts faithfully on $S^{1}$ as described in $\$ 1.6$. (This action was originally observed by Odden [Odd05, Proposition 4.5].) Since any square and any commutator in $\operatorname{Homeo}\left(S^{1}\right)$ is orientation preserving, we see that $\left[\operatorname{Homeo}\left(S^{1}\right): \mathrm{Homeo}_{+}\left(S^{1}\right)\right]=2$. Moreover, all finite subgroups of $\mathrm{Homeo}_{+}\left(S^{1}\right)$ are cyclic [BS18, Lemma 3.1], it follows that every finite subgroup of $\operatorname{Comm}\left(\Gamma_{2}\right)$ contains a cyclic subgroup of index at most 2. 


\section{A parting Proof With Questions}

6.1. If a finitely-generated group $\Gamma$ has solvable word problem, then finitelygenerated subgroups of $\operatorname{Aut}(\Gamma)$ have solvable word problem. The same is true of $\operatorname{Comm}(\Gamma)$ when $\Gamma$ is finitely presented and satisfies the unique root property. In particular, finitely-generated subgroups of abstract commensurators of surface groups have solvable word problem.

Proposition 6. Let $\Gamma$ be a finitely presented group with solvable word problem and the unique root property. Then any finitely generated subgroup of $\operatorname{Comm}(\Gamma)$ has solvable word problem.

Proof. Fix a finite set $S$ of elements in $\operatorname{Comm}(\Gamma)$. We outline an algorithm that inputs a word in $S$ and outputs "True" if the word is the identity, and "False" otherwise. We take as constants in our algorithm representatives for each element in $S$, along with indices and finite generating sets for each of the domains and codomains of elements in $S$ (such generating sets exist by the Reidemeister-Schreier Method). Given the above constants, the inverse of each element in $S$ can be computed, so without loss of generality we assume that $S$ is symmetric.

We start with the simplest case, when the word is of length one. Let $g: A \rightarrow$ $B$ be the representative of the commensurator of $\Gamma$ corresponding to the letter, where $A, B$ are of finite index inside $\Gamma$ and have explicitly written generating sets. The isomorphism $g$ is fully determined by its values on the fixed finite generating set for $A$. Since the word problem in $\Gamma$ is solvable, determining whether $g$ fixes each generator of $A$ is solvable, and hence determining whether $g$ is the identity is solvable. It follows, by our assumptions on $\Gamma$, that determining whether the commensurator induced by $g$ is the identity is solvable.

For a word of length two we have two maps $g: A \rightarrow B$ and $h: C \rightarrow D$, where $A, B, C, D$ are all of finite index inside $\Gamma$ and have explicitly written generating sets and indices. Define

$$
g \star h=\left.\left.g\right|_{A \cap D} \circ h\right|_{h^{-1}(A \cap D)}
$$

which is a map $h^{-1}(A \cap D) \rightarrow g(A \cap D)$ representing the composition of commensurators $[g] \circ[h] \in \operatorname{Comm}(\Gamma)$. Because $\Gamma$ has the unique root property, $[g] \circ[h]$ is trivial in $\operatorname{Comm}(\Gamma)$ if and only if $g \star h$ is the identity function. Since $\Gamma$ is finitely presented, there is an algorithm for finding a finite quotient of $\Gamma$ whose kernel is contained in $A \cap D$. Thus, there is an algorithm for computing a finite generating set for $A \cap D$, and subsequently $h^{-1}(A \cap D)$. As the homomorphism $g \star h$ is determined by its values on a generating set of $h^{-1}(A \cap D)$, determining whether $g \star h$ is the identity is solvable because $\Gamma$ has solvable word problem.

Repeating this process inductively for arbitrary compositions $h_{1} \star h_{2} \star h_{3} \star \cdots \star h_{k}$ for letters $h_{i}$, allows one to handle word of arbitrarily length, giving the desired algorithm.

6.2. The above proposition motivates the following questions:

(1) Which groups $\operatorname{Comm}(\Gamma)$ from Proposition 6 contain a copy of a nonresidually finite Baumslag-Solitar group?

(2) Does there exist a torsion-free group $G$ that embeds inside $\operatorname{Comm}\left(F_{2}\right)$ but not inside $\operatorname{Comm}\left(\Gamma_{2}\right)$ ? Vice versa?

(3) Let $\Gamma$ be a surface group. Do finitely generated subgroups of $\operatorname{Comm}(\Gamma)$ satisfy a Tits' alternative? 
Note that a partial answer to Question 3 is given in the compact surface group case by [Mar00, using the fact that $\operatorname{Comm}(\Gamma)$ embeds in $\operatorname{Homeo}\left(S^{1}\right)$ Odd05].

\section{Appendix A. The GAP System}

In the appendices that follow we use the GAP System to complete our proofs. Borrowing words from the creators GAP17: "GAP is a system for computational discrete algebra, with particular emphasis on Computational Group Theory. GAP provides a programming language, a library of thousands of functions implementing algebraic algorithms written in the GAP language as well as large data libraries of algebraic objects." GAP is especially well-suited for our needs, with key functions that we will use to explicitly evaluate commensurators. Here is a short glossary of some of the key functions used in our code:

GroupHomomorphismByImages( domain, codomain, list1, list2 ). Inputs two groups "domain" and "codomain" with lists "list1" and "list2". When GAP runs this command it first verifies that a well-defined homomorphism from "domain" to "codomain" sending "list1" to "list2" exists, returning "fail" otherwise. If the homomorphism requested is well-defined, this returns a homomorphism from domain to codomain where elements of "list1" consisting of generators of domain are sent to corresponding elements in "list2".

Image(map, elem). Inputs a homomorphism "map" and an element from the domain "elem". Returns the image of element "elem" under an application of the homomorphism "map".

InverseGeneralMapping(map). Inputs a homomomorphism "map". This function returns an inverse of an isomorphism of groups where the domain and codomain are not equal.

IsomorphismSimplifiedFpGroup $(\mathrm{G})$. Inputs a finitely presented group "G", for which GAP applies Tietze transformations to a copy in order to reduce it with respect to the number of generators, the number of relators, and the relator lengths. When we apply this to a finite-index subgroup of an oriented cocompact surface group we get a one-relator group, as expected. This function returns an isomorphism with domain $\mathrm{G}$, codomain a group $\mathrm{H}$ isomorphic to $\mathrm{G}$, so that the presentation of $\mathrm{H}$ has been simplified using Tietze transformations.

IsOne(elem). Inputs an element "elem" from a group. Returns true if "elem" is equal to the identity, and false if "elem" is not. This function is not guaranteed to terminate.

\section{Appendix B. Computer-Assistance for the free group Case}

The code in $\$ \mathrm{~B} .1$ concretely defines maps $\phi$ and $\psi$ from the proof of the free group case. Here K1 and K2 are the subgroups $\Delta_{1}$ and $\Delta_{2}$, and K1.1 corresponds to the element $A^{-2}$ in the free group $\langle A, B\rangle$.

Running this code outputs "false". The GAP code verifies that each isomorphism exists and that the domains and ranges are all appropriate. In the end the GAP code computes the function

$$
w=\phi^{-1} \psi \phi \psi^{-1} \phi^{-1} \psi \phi \psi^{-1} \phi^{-1}
$$


with input Word $=B A B^{-1} A^{-1}$. It outputs Word10 $=A^{3} B A B^{-1} A^{2}$, and verifies that these are not equal (although this last part is easily done by hand).

After running the code below one can investigate properties of the map $\psi$. For instance, here $\psi$ is evaluated on $A^{-2}$ :

gap> K1.1;

$A^{-}-2$

gap> Image(psi, K1.1);

$A^{\wedge}-3$

The variable K1.1, corresponding to the element $A^{-2}$ in $F_{2}$, is shown here as being mapped to $A^{-3}$.

Moreover, one can verify that psi is an isomorphism K1 $\rightarrow$ K2 in GAP by checking that the map is surjective and that K1 and K2 have the same index in f:

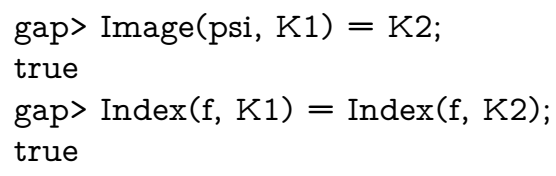

B.1. The code. We ran the following code on GAP version 4.8.8. The usefulness of the code below rests on the identifications of the generator K1.1 with $A^{-2}$ and the generator K2.2 with $A^{3}$. Be aware that other versions of GAP may have different implementations of the function Kernel, in which $A^{2}$ and $A^{3}$ may correspond to different generators, or may not even be part of the chosen free generating set.

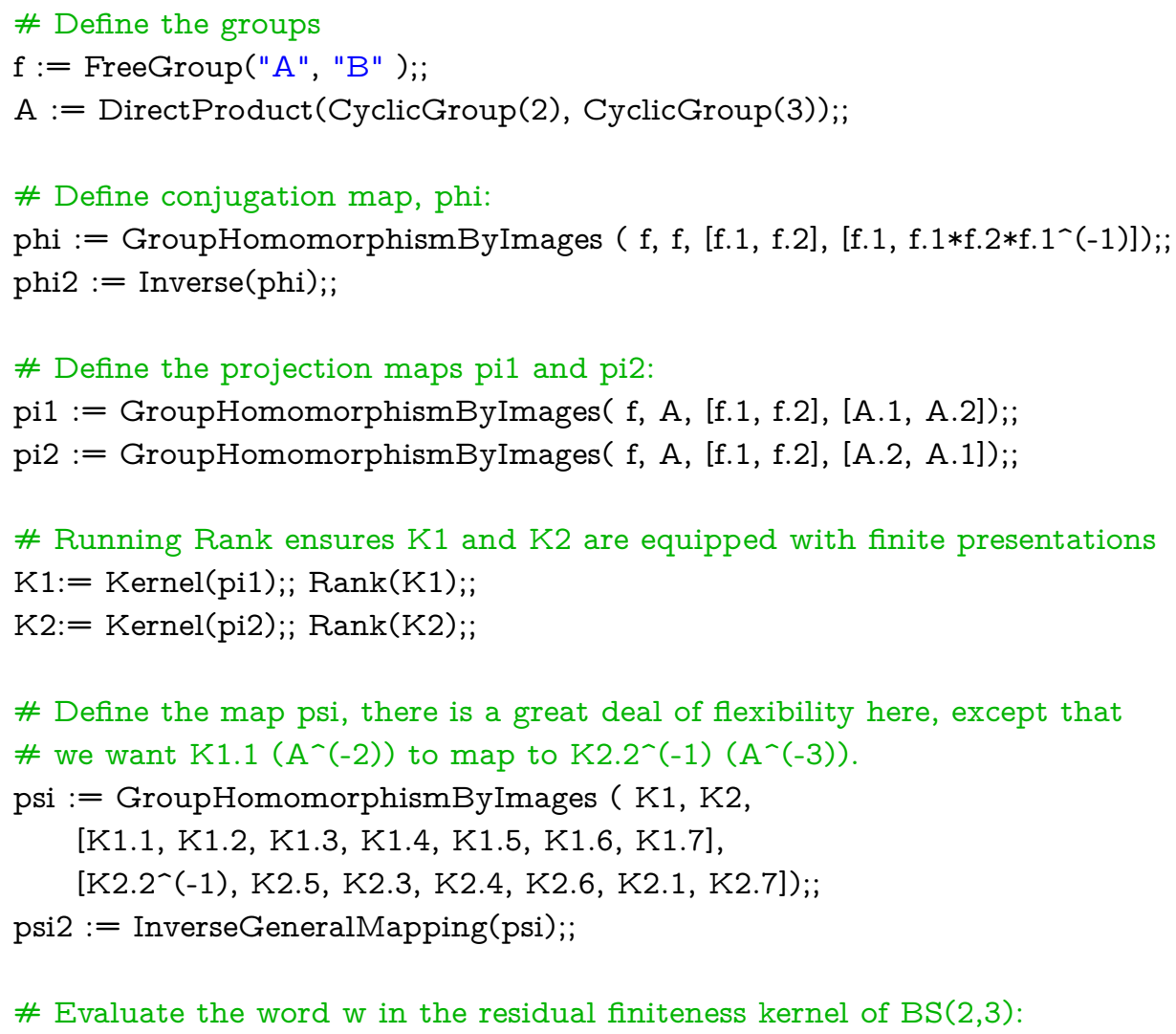




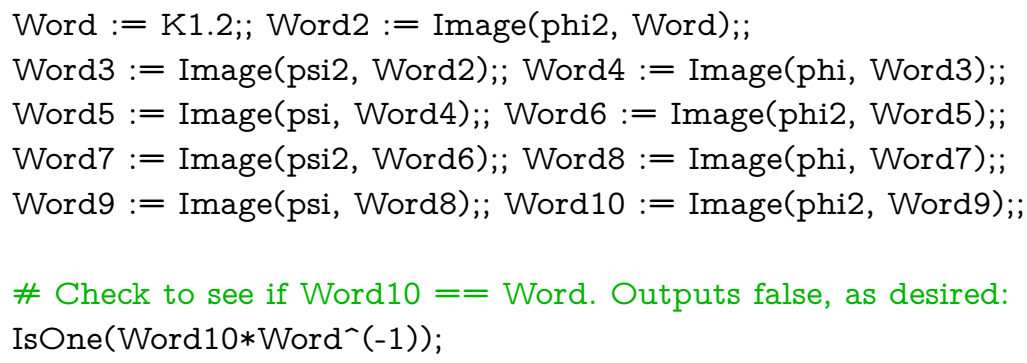

\section{Appendix C. Computer-Assistance for the cocompact Case}

The code in $\S$ C.1 concretely defines maps $\phi$ and $\psi$ from the proof of the cocompact Fuchsian case. Here K1 and K2 are the subgroups $\Delta_{1}$ and $\Delta_{2}$, and K1.1 corresponds to the element $A^{-2}$ in the surface group $g=\langle A, B, C, D:[A, B][C, D]\rangle$.

In this case, more care must be taken in defining the map psi : K1 $\rightarrow \mathrm{K} 2$ because $\mathrm{K} 1$ and K2 are not free groups. Explicitly defining maps from K1 to K2 using the GAP function GroupHomomorphismByImages usually results in maps that GAP cannot verify are well-defined isomorphisms (moreover, finding such maps from scratch is prohibitively difficult). To get around this obstruction, we first simplify the presentations of $\mathrm{K} 1$ and $\mathrm{K} 2$ before finding an isomorphism (akin to diagonalizing a matrix before doing computations).

We briefly explain the construction of psi in the code now. First, the code defines group homomorphisms Iso1 : K1 $\rightarrow \mathrm{fp} 1$, Hom1 : fp1 $\rightarrow$ Image1, Iso2 : K1 $\rightarrow \mathrm{fp} 2$, and Hom2 : fp2 $\rightarrow$ Image2. GAP does not view $\mathrm{K} 1$ and $\mathrm{K} 2$ as finitely presented groups, and so the maps Iso1 and Iso2 are simply isomorphisms to their finite presentations (computed from the fact that $\mathrm{K} 1$ and $\mathrm{K} 2$ are finite-index normal subgroups). The maps Hom1 and Hom2 are maps to simplified finite presentations. The resulting maps from this construction are version dependent, so running our code in a different version of GAP may result in lastMap not being well-defined (in which case GAP will throw a fault). The images of the maps Hom1 and Hom2 have the following presentations. The red color indicates a part of the relation that is significantly different from the rest.

$$
\begin{aligned}
\text { Image1 }= & \left\langle F_{1}, F_{2}, F_{3}, F_{4}, F_{5}, F_{6}, F_{7}, F_{8}, F_{9}, F_{10}, F_{11}, F_{12}, F_{13}, F_{14}:\right. \\
& {\left.\left[F_{11}, F_{12}\right]\left[F_{4}, F_{5}\right] F_{1}^{-1} F_{6} F_{1}\left[F_{13}, F_{14}\right]\left[F_{9}, F_{10}\right] F_{6}^{-1}\left[F_{7}, F_{8}\right]\left[F_{2}, F_{3}\right]=1\right\rangle, } \\
\text { Image2 }= & \left\langle F_{1}, F_{2}, F_{3}, F_{4}, F_{5}, F_{6}, F_{7}, F_{8}, F_{9}, F_{10}, F_{11}, F_{12}, F_{13}, F_{14}:\right. \\
& {\left.\left[F_{5}, F_{6}\right] F_{4}\left[F_{13}, F_{14}\right]\left[F_{7}, F_{8}\right] F_{1}^{-1} F_{4}^{-1} F_{1}\left[F_{9}, F_{10}\right]\left[F_{2}, F_{3}\right]\left[F_{11}, F_{12}\right]=1\right\rangle . }
\end{aligned}
$$

The image of $A^{-2}$ under Hom1 $\circ$ Iso1 is $F_{1}$ in Image1. Moreover, $F_{4}$ maps to $A^{3}$ under (Hom2 $\circ$ Iso2) ${ }^{-1}$. Then lastMap : Image $\rightarrow$ Image 2 is defined to be an isomorphism taking $F_{1}$ to $F_{4}$. The resulting map psi : $\mathrm{K} 1 \rightarrow \mathrm{K} 2$ is then defined by the composition

$$
\text { psi }:=\text { flipHom } \circ \text { Iso } 2^{-1} \circ \text { Hom } 2^{-1} \circ \text { lastMap } \circ \text { Hom } 1 \circ \mathrm{Iso} 1,
$$

where flipHom sends $A$ to $A^{-1}$, correcting that lastMap sends $A^{-2}$ to $A^{3}$.

After verifying that the map psi is well-defined, the computer uses the finite presentation to verify that the input and output of the function

$$
w=\phi^{-1} \psi \phi \psi^{-1} \phi^{-1} \psi \phi \psi^{-1} \phi^{-1}
$$


are not identical when evaluated at $\mathrm{K} 1.2=\mathrm{C}$ in $g$ (one can also do this last check by hand using Dehn's algorithm). The output to this evaluation, Word10, is:

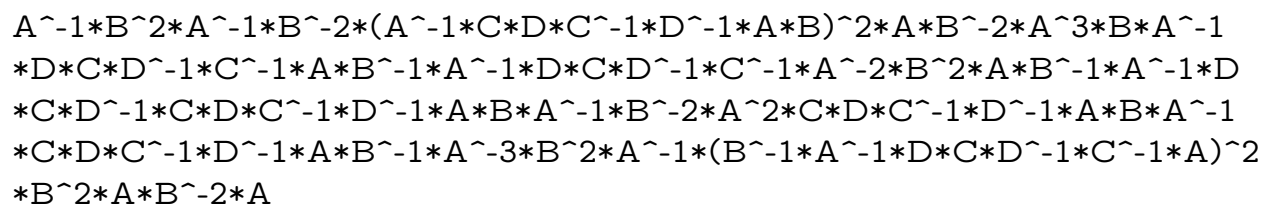

As before, K1.1 corresponds to $A^{-2}$ and applying $\psi$ to it yields $A^{-3}$, as desired: gap> K1.1;

$A^{\wedge}-2$

gap> Image(psi, K1.1);

$A^{\wedge}-3$

Moreover, one can verify that psi is an isomorphism K1 $\rightarrow$ K2 in GAP by checking that the map is surjective and that $\mathrm{K} 1$ and $\mathrm{K} 2$ have the same index in g:

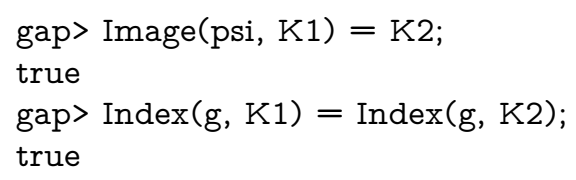

C.1. The code. We ran the following code on GAP version 4.8.8. Note again that different versions of GAP may have different implementations of key functions, such as IsomorphismFpGroup and IsomorphismSimplifiedFpGroup, which are used below to find explicit presentations of $\Delta_{1}$ and $\Delta_{2}$. Because the definition of $\psi$ uses the presentations output by these functions, the definition below may not determine an isomorphism in other versions of GAP.

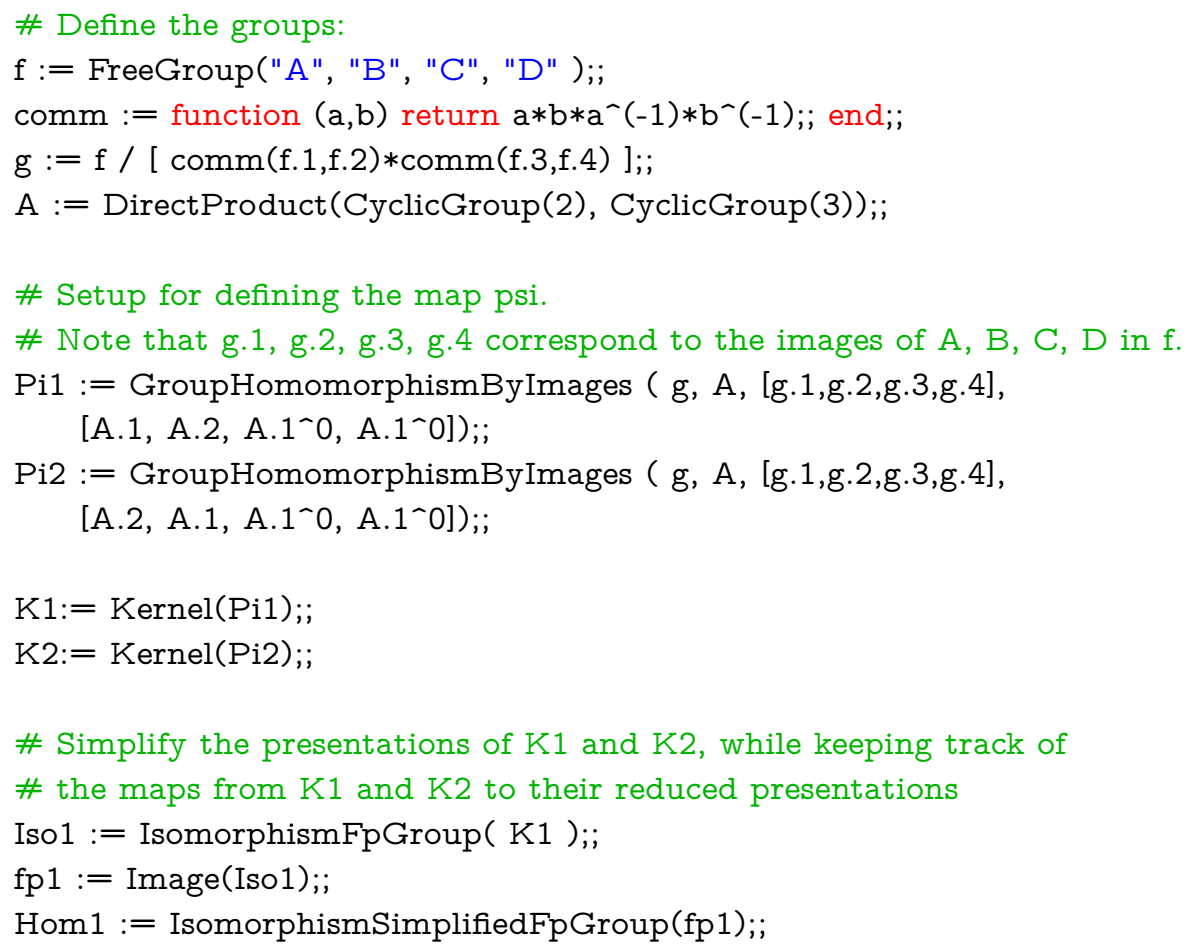




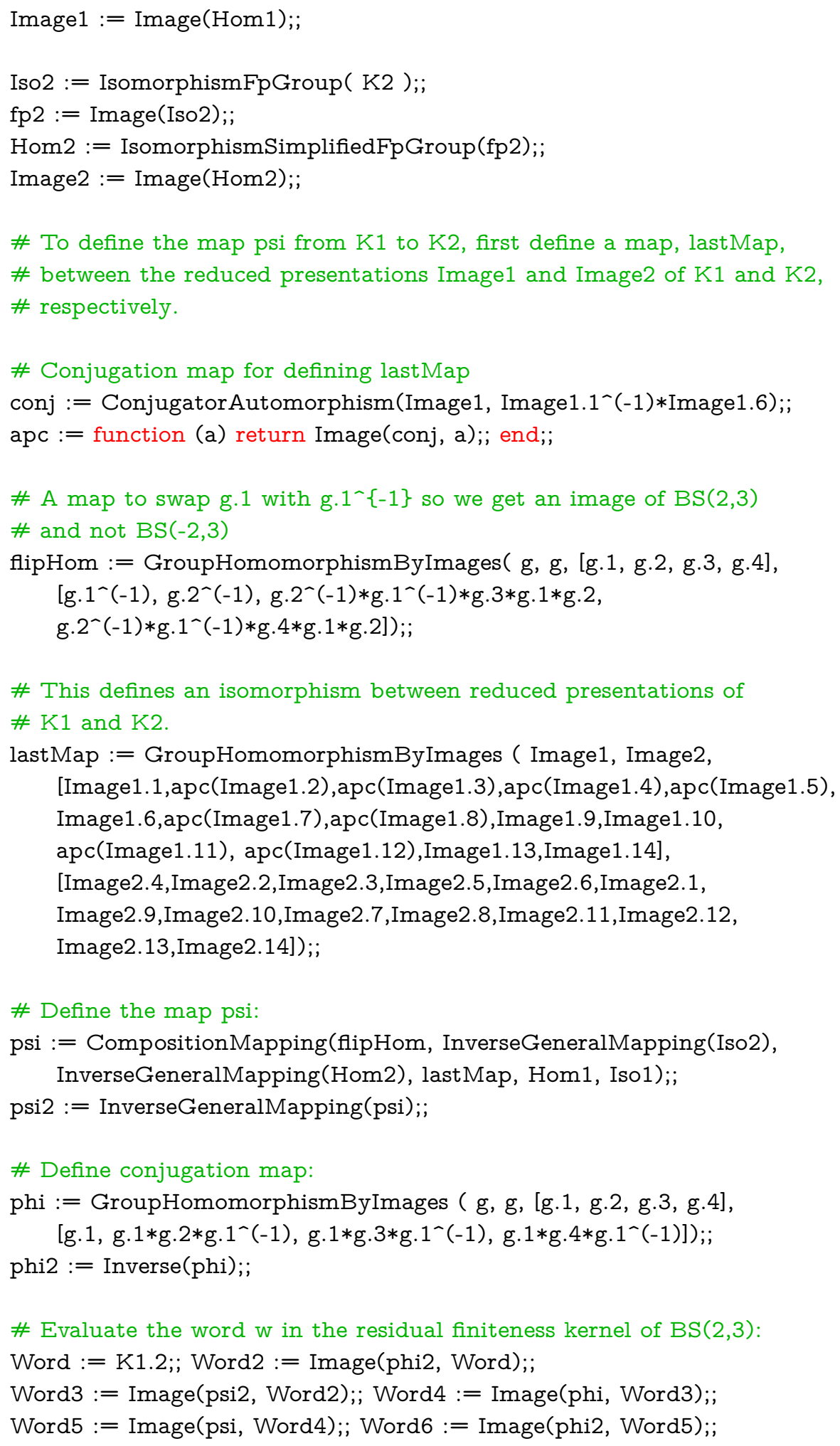




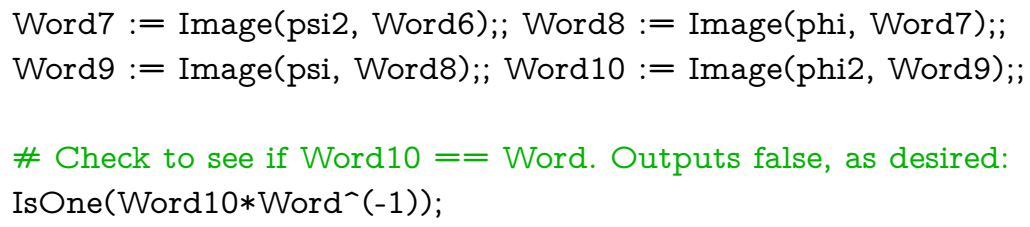

\section{REFERENCES}

[Bau63] Gilbert Baumslag, Automorphism groups of residually finite groups, J. London Math. Soc. 38 (1963), 117-118. MR 0146271

[BB10] L. Bartholdi and O. Bogopolski, On abstract commensurators of groups, J. Group Theory 13 (2010), no. 6, 903-922. MR 2736164

[BH99] Martin R. Bridson and André Haefliger, Metric spaces of non-positive curvature, Grundlehren der Mathematischen Wissenschaften [Fundamental Principles of Mathematical Sciences], vol. 319, Springer-Verlag, Berlin, 1999. MR 1744486

[BM97] Marc Burger and Shahar Mozes, Finitely presented simple groups and products of trees, C. R. Acad. Sci. Paris Sér. I Math. 324 (1997), no. 7, 747-752. MR 1446574

[Bor60] Armand Borel, Density properties for certain subgroups of semi-simple groups without compact components, Ann. of Math. (2) 72 (1960), 179-188. MR 0123639

[Bri63] John L. Britton, The word problem, Ann. of Math. (2) 77 (1963), 16-32. MR 0168633

[BS62] Gilbert Baumslag and Donald Solitar, Some two-generator one-relator non-Hopfian groups, Bull. Amer. Math. Soc. 68 (1962), 199-201. MR 0142635

[BS18] Hyungryul Baik and Eric Samperton, Spaces of invariant circular orders of groups, Groups Geom. Dyn. 12 (2018), no. 2, 721-763. MR 3813208

[FH07] Benson Farb and Michael Handel, Commensurations of Out $\left(\mathrm{F}_{n}\right)$, Publ. Math. Inst. Hautes Études Sci. (2007), no. 105, 1-48. MR 2354204

[FM02] B. Farb and L. Mosher, The geometry of surface-by-free groups, Geom. Funct. Anal. 12 (2002), no. 5, 915-963. MR 1937831

[FM12] Benson Farb and Dan Margalit, A primer on mapping class groups, Princeton Mathematical Series, vol. 49, Princeton University Press, Princeton, NJ, 2012. MR 2850125

[GAP17] The GAP Group, GAP - Groups, Algorithms, and Programming, Version 4.8.8, 2017.

[Gro75] Edna K. Grossman, On the residual finiteness of certain mapping class groups, J. London Math. Soc. (2) 9 (1974/75), 160-164. MR 0405423

[Mal56] A. L. Mal'cev, On certain classes of infinite solvable groups, Amer. Math. Soc. Transl. (2) 2 (1956), 1-21. MR 0075950 (17,824d)

[Mal71] Anatolii Ivanovic Malćev, The metamathematics of algebraic systems. Collected papers: 1936-1967, North-Holland Publishing Co., Amsterdam-London, 1971, Translated, edited, and provided with supplementary notes by Benjamin Franklin Wells, III, Studies in Logic and the Foundations of Mathematics, Vol. 66. MR 0349383

[Man87] A. Mann, Problems about automorphisms of infinite groups, Second Intn'l Conf Group Theory (1987), Debrecen.

[Mar91] G. A. Margulis, Discrete subgroups of semisimple Lie groups, Ergebnisse der Mathematik und ihrer Grenzgebiete (3) [Results in Mathematics and Related Areas (3)], vol. 17, Springer-Verlag, Berlin, 1991. MR 1090825

[Mar00] Gregory Margulis, Free subgroups of the homeomorphism group of the circle, C. R. Acad. Sci. Paris Sér. I Math. 331 (2000), no. 9, 669-674. MR 1797749

[Odd05] Chris Odden, The baseleaf preserving mapping class group of the universal hyperbolic solenoid, Trans. Amer. Math. Soc. 357 (2005), no. 5, 1829-1858. MR 2115078

[R0̈2] Claas E. Röver, Abstract commensurators of groups acting on rooted trees, Proceedings of the Conference on Geometric and Combinatorial Group Theory, Part I (Haifa, 2000), vol. 94, 2002, pp. 45-61. MR 1950873

[Sha00] Yehuda Shalom, Rigidity of commensurators and irreducible lattices, Invent. Math. 141 (2000), no. 1, 1-54. MR 1767270

[Stu15] Daniel Studenmund, Abstract commensurators of lattices in Lie groups, Comment. Math. Helv. 90 (2015), no. 2, 287-323. MR 3351746 
Department of Mathematics, The City College of New York

E-mail address: khalid.math@gmail.com

UNIVERSity OF Notre DAME

E-mail address: dstudenm@nd.edu 